\title{
La educación como objeto de interés para las ciencias de la complejidad
}

\author{
The education as a subject of interest \\ of the science of complexity
}

Cecilia Dimaté Rodríguez*

Resumen

Las ciencias de la complejidad se consideran hoy el punto más avanzado del estudio de las ciencias mismas, en tanto, con el desarrollo de sus temas se busca transformar las relaciones entre los seres humanos y su entorno en general, bajo el espectro de una construcción teórica enraizada en una visión multidisciplinar. La educación, como campo de producción y reproducción de saber, debería configurar también un objeto de estudio de las ciencias de la complejidad, sin embargo, las aproximaciones que desde las ciencias de la complejidad se han hecho al estudio de la educación han sido escasas y se han centrado esencialmente en una de sus corrientes denominada pensamiento complejo. El presente artículo aborda la temática de la educación en el marco de las ciencias de la complejidad que, necesariamente, lleva a la reflexión del papel que estas ciencias cumplen actualmente en la configuración del tema educativo.

\section{Palabras clave:}

Complejidad, pensamiento complejo, educación.

\section{Abstract}

Today, sciences of the complexity are considered the most advanced point of the study of sciences themselves. Through the development of their subjects, each science searches to transform the relations between human beings and their general surroundings. This is done under the view of a theoretical construction deeply rooted in a multidisciplinar vision. The education as a field of production and reproduction of knowledge should also form part of the areas of study of sciences of the complexity. Nevertheless, the approaches from the sciences of the complexity geared towards the study of the education have been few and they have been centered essentially in one of its approaches denominated "complex thought". The following article tackles the education thematic in the framework of the sciences of the complexity that, necessarily, takes reflection of the role that is now used by these sciences to accomplish the configuration of the educational area.

\section{Keywords:}

Complexity, complex thinking, education

Artículo recibido el 29 de julio de 2007 y aprobado el 31 de octubre de 2007

* Profesora del Departamento de Lenguas, Universidad Pedagógica Nacional. Integrante del Grupo de Investigación en Pedagogía, Comunicación y Lenguaje (GIPELEC). Profesora de la Facultad de Educación de la Universidad Externado de Colombia. Estudiante del Doctorado en Estudios Políticos de la Universidad Externado de Colombia. cdimate@pedagogica.edu.co 
Aproximarse al campo de las ciencias de la complejidad y, a partir de él, ubicar lo que ha sido la educación para estas nuevas visiones de la ciencia, implica un arduo trabajo de revisión de la literatura producida al respecto $y$, fundamentalmente, un enorme trabajo de comprensión de lo que subyace -en términos de la construcción teórica de las ciencias mismas- a la explicación de lo que significaría entender la educación como un fenómeno complejo.

No es propósito de este trabajo agotar las dos tareas enunciadas, especialmente, porque: a) aunque el avance alcanzado en las ciencias de la complejidad ha permitido una mayor producción académica, no tanto así ha ocurrido con el tema educativo en dicho marco, lo cual significa que la producción al respecto, realmente es escasa; y b) aunque, como lo advierte Maldonado (2005, p. 10), existe "un ávido deseo de estudios sobre la complejidad, [también existen] limitaciones de tipo lingüístico, o de formación" que se convierten en obstáculo para alcanzar una mayor comprensión del tema desde el nivel esencialmente científico, máxime cuando no se encuentra mayor producción en el campo teórico de la relación ciencias de la complejidad y educación. Pese a esto, la revisión que se registra en estas líneas aspira a ofrecer un panorama general de lo que ha sido el estudio de la educación en el marco de las ciencias de la complejidad, especialmente, en el ámbito conceptual desde el cual se ha alcanzado un mayor desarrollo: el pensamiento complejo.

Así las cosas, la primera parte del artículo comprende un sucinto recorrido por la evolución de las ciencias de la complejidad, recorrido que permite además de la ubicación en el tema, el abrebocas para plantear el abordaje de la educación en dicho marco. En la segunda parte se hace un acercamiento al concepto de complejidad y al camino que toma en lo que se refiere a la educación. Finalmente, y como propósito fundamental del tema que interesa, se presentarán algunos de los desarrollos en educación en cuanto a lo que se conoce como pensamiento complejo.

\section{Las ciencias de la complejidad: acercamiento a su evolución}

En la medida en que este documento no constituye una exhaustiva revisión del tema de las ciencias de la complejidad y que esta primera parte sólo pretende una ubicación en el tema, cabe anotar que en la mayoría de los textos publicados al respecto durante las dos últimas décadas es recurrente encontrar una referencia al origen y desarrollo de estas ciencias previo al abordaje del tema que interesa a cada autor; recorrido que se desarrolla en mayor o menor profundidad y detalle según los propósitos que guían la publicación respectiva.

Así, por ejemplo, uno de los textos que refieren ya un primer esbozo sobre el origen y los desarrollos iniciales de lo que actualmente se agrupa bajo la denominación de ciencias de la complejidad, es el de Carreras, Escorihuela y Requejo, editores del libro Azar, caos e indeterminismo, que se publica en el año 1990, pero cuyo contenido tiene como primer referente un curso ofrecido en 1987 en la Universidad de Verano de Teruel sobre "Azar y determinismo" y su primera divulgación en el Seminario Interdisciplinar de la Universidad de Zaragoza, en 1988. El trabajo registrado en esta publicación es "sobre todo de recopilación de teorías y esclarecimiento de ideas [y un] artículo introductorio, con dos finalidades 'establecer un marco especulativo general y centrar[se] en el campo de las matemáticas, patrimonio común de las diversas ciencias'" (1990, p. 8). El trabajo de este equipo de profesores comparte, entonces, el hecho de encarar la discusión sobre conceptos básicos y teorías de las recientes $\mathrm{y}$-en ese entonces- poco conocidas ciencias de la complejidad: así, caos, azar, indeterminismo, incertidumbre, catástrofes, estructuras disipativas, fractales, atractores, entre otros, son los conceptos abordados con la particularidad que cada uno de los autores los refiere a un dominio específico de su saber: física, matemáticas, filosofía, economía.

Otro texto que se publica en francés a inicios de la década de los noventa (en su versión al español, en 1996) es la Introducción al pensamiento complejo de 
Edgar Morin, publicación que se configura en uno de los documentos sobre el enfoque de complejidad quizás más desarrollado en el campo educativo. El prólogo del texto elaborado por el mismos autor, refiere los antecedentes de su concepción y el trabajo que él ha desarrollado respecto a la complejidad, desde sus primeras publicaciones, cuya palabra aparece en sus escritos, según él, a finales de 1960 "vehiculizada por la teoría de la Información, la cibernética, la teoría de sistemas, el concepto de autoorganización" (1996, p. 24) y cuya formación y desarrollo se configura mejor en sus publicaciones posteriores: el Método, en el año 1970; El paradigma perdido, en 1973; y Más allá de la complicación, la complejidad, incluido en la primera edición de la Ciencia en conciencia (Morin, 1996, p. 24). Esta publicación está organizada en seis capítulos, pero es en el segundo en el que el autor refiere con más detalle el origen y desarrollo de la idea de complejidad, asignándole a Wiener y Ashby, los fundadores de la cibernética, la entrada de esta idea en la escena de la ciencia y a Von Neumann el concepto de complejidad enlazado con los fenómenos de autoorganización (Morin, 1996, p.59). Años más tarde (1999), Morin publica L'Intelligence de la complexité, artículo en el que desarrolla su idea de una epistemología de la complejidad y en el que plantea como su problemática central el suficiente desarrollo de la competencia del individuo "para articularla con otras competencias, la cuales, encadenadas, formarían un bucle completo y dinámico, el bucle del conocimiento del conocimiento" (Morin, 2004, p. 19). La obra de Morin ha sido prolífica en el tema y su importancia para la educación ha sido significativa, pues podría decirse que es el punto de partida para el estudio y transformación de la concepción de educación en los últimos tiempos.

Colombia no ha escapado al interés por el tema y, para los efectos de la presente revisión, se podría decir que hay dos trabajos que sirven de orientación para quienes incursionan en este campo en nuestro país: el Manual de iniciación pedagógica al pensamiento complejo, publicado por la Corporación para el Desarrollo Complexus, el ICFES y UNESCO en 2002 y la extensa producción del profesor CARLOS EdUARDO MALDONADO quien, a través de diversas universidades del país ha procurado la difusión y el conocimiento de lo que son las ciencias de la complejidad.

Respecto del Manual de iniciación pedagógica al pensamiento complejo, cabe decir que aunque la publicación tiene un profundo espíritu moriniano, ya que su elaboración se produce en el marco de las visitas que Morin hizo a Colombia, el documento, especialmente en su primera parte, constituye un detallado análisis por lo que ha sido la evolución de la complejidad en el mundo de las ciencias, aportando desde conceptos básicos hasta autores e hitos de su transformación.

En relación con el trabajo de Maldonado, como ya se dijo la producción es extensa y, además, tiene la particularidad de que en ella es posible identificar la idea de ciencias de la complejidad que escapa a buena parte de las publicaciones referidas anteriormente. Maldonado no se detiene en el caos, en la teoría de las catástrofes o en la termodinámica, sino que presenta las ciencias de la complejidad en su origen, en su trayectoria y en su carácter de ciencias cuya reflexión se encuentra. Alrededor de los temas de irreversibilidad, de los procesos de organización -léase autoorganización- y de innovación como cabe construir las teorías que habrán de transformar -como efectivamente es el caso- a las ciencias, esto es, al mundo. Pero con ellas, lo que es aún más significativo, [...] sobre los temas que habrán de transformar radicalmente las relaciones entre los seres humanos, entre el hombre y la naturaleza, en fin, a la sociedad en general (Maldonado, 2005, p. 40).

Como quiera que este autor ha trabajado intensamente el tema, es posible encontrar en varios de sus escritos aspectos relacionados con el origen y desarrollo de las ciencias de la complejidad, tal es el caso de, entre otros:

El libro Visiones sobre la complejidad (1999), cuya edición está bajo su responsabilidad y en el cual se encuentra un artículo de su autoría en el que se aborda el tema de la complejidad en el marco de la filosofía de la ciencia. El texto en mención ofrece una caracterización de lo que él denomina la lógica de la complejidad, centrando su atención, especialmente, en lo que sería la complejidad como ciencia, camino que le permite allanar posteriormente una 
filosofía de la lógica de la complejidad (Maldonado, 1999, pp. 12-13).

El artículo "Ciencias de la complejidad: ciencias de los cambios súbitos", publicado en 2005, año de publicación de dos libros en los que el autor profundiza en el tema de las ciencias de la complejidad desde una perspectiva más teórica, más desde la filosofía de la ciencia. En el artículo se encuentra una exposición bastante clarificadora, por cierto, de lo que son las ciencias de la complejidad, su origen, su cronología y algunos de los conceptos básicos.

El libro Termodinámica de la complejidad, publicado en el mismo año del artículo anterior y que constituye un amplio desarrollo de lo que -en resumen, según el mismo autor- se encuentra en el artículo anterior, presenta además del origen, la trayectoria y los conceptos de las ciencias de la complejidad, una extensa discusión acerca de lo que son estas ciencias y de su papel en el marco de la transformación de la ciencia en los últimos años; pero, fundamentalmente es "una introducción a la complejidad para las ciencias sociales y humanas" (Maldonado, 2005, p. 12). Con esta directriz, el texto tiene su fundamento en la obra de Prigogine, la figura más destacada del estudio de la termodinámica y que, para casi todos los autores referidos en la presente revisión, se considera como el padre de la complejidad.

Un tercer libro que aporta en términos del origen y de la evolución de las ciencias de la complejidad, compilado por Maldonado (2005a), es Complejidad de las ciencias y ciencias de la complejidad, en el que la discusión se centra en la medición, como "uno de los problemas centrales de la complejidad" (MALDONADO, 2005a, p. 19) pero que, necesariamente incluye un estudio -no tan extenso como en la otra publicación- sobre la complejidad. La publicación recoge también la discusión acerca del tema tratado -sea de manera teórica o práctica- en el centro de algunas disciplinas: química, física, biología.

\section{Complejidad: algunas definiciones}

Esta segunda parte de la revisión, pretende dar cuenta de algunos de las definiciones de complejidad que se han utilizado con más frecuencia y que sirven de referente para entrar al tema de la educación. Sin embargo, cabe anotar que, al ser las ciencias de la complejidad un terreno en construcción y para hacer honor a los aspectos centrales que la constituyen, resulta indispensable aclarar que son varios los conceptos que se han venido trabajando alrededor de dichas ciencias. Como puede verse en los dos textos introductorios al tema de la complejidad y las ciencias sociales, elaborados por Maldonado, cada una de las diferentes ciencias de la complejidad construye sus propios conceptos teniendo como base para estas ciencias en plural que "el estudio de la complejidad consiste, dicho de un modo básico, en el estudio de la dinámica no lineal" (Maldonado, 2005a, p. 92).

Así, para la termodinámica del no-equilibrio la irreversibilidad, las estructuras disipativas y el tiempo (la flecha) serían los conceptos fundamentales; para las ciencias del caos lo serían la turbulencia y los atractores; la geometría de fractales hablaría de azar y escalantes (o autosimilitud, como posteriormente se denominó); en la teoría de las catástrofes el campo de estudio se concentra en el concepto de discontinuidades y catástrofes; $y$, finalmente, para las lógicas no clásicas, las cuales precisamente encaran aquellas lógicas abandonadas por la lógica formal clásica estarían conceptos como: paraconsistencia, relevancia, modalidades, polivalencias, temporalidades y cuántica. Como lo dice el mismo Maldonado (2005a, p. 114) existen múltiples conceptos constitutivos de la complejidad, sin embargo, con el riesgo de la exclusión los más destacados son: autoorganización, emergencia, no-linealidad, irreversibilidad, tiempo y temporalidad, estructuras disipativas, recursividad y bucles de retroalimentación.

Siendo el propósito de este documento, la revisión del tema que da cuenta de la relación complejidad y educación, a continuación se hará una revisión solamente de las definiciones de complejidad de mayor uso en el terreno de esta relación, en la medida en que es el punto central y abarcador de las producciones que se han dado alrededor de la temática.

Para la totalidad de los autores estudiados en esta revisión, es común afirmar que no existe una única definición de complejidad y de la misma manera en 
que cada uno lo afirma, también plantea un punto de referencia de la definición para poder desarrollar el estudio que le interesa. Entre estos autores cabe destacar un artículo elaborado por Binder, en el que hace un recorrido por el concepto desde varios puntos de vista: lenguajes, información, posmodernismo y mundo real. Con estos cuatro referentes, los marcos teóricos de la complejidad varían, según él, “en el tipo de bases teóricas que invocan, el papel del observador, su diferencia con los paradigmas actuales y la universalidad de su aplicación. Algo común entre los cuatro es el énfasis en la información" (1999, p. 47).A continuación se presentan algunas definiciones más específicas encontradas en documentos que o bien enmarcan su trabajo en el campo de las ciencias de la complejidad en general o lo delimitan para el terreno específico de la educación:

La definición en Maldonado se ubica en el marco de las ciencias de la complejidad y es allí donde origina una definición de complejidad asociada a los sistemas:

[Fenómenos, comportamientos o sistemas] que están marcados por inestabilidades, fluctuaciones, sinergia, emergencia, autoorganización, no-linealidad, bucles de retroalimentación positiva antes que de retroalimentación negativa, equilibrios dinámicos, rupturas de simetría, en fin, aquellos que se encuentran en el filo del caos (Maldonado, 2005, p. 89).

Sin ser un concepto definido en su momento, Prigogine alude al tema como la tarea resultante de lo que serán posteriormente las ciencias físicas.

Las ciencias físicas están inmersas en un proceso de reconceptualización, y es significativo que éste se haya iniciado en un marco que, a partir de la explosión demográfica ( $\mathrm{y}$ de otros aspectos sociales, como el auge experimentado por las técnicas informáticas) nos llevará más tarde o temprano al desmoronamiento de los conceptos a veces simplistas con los que se pretendía describir las sociedades humanas.

Reconocer la complejidad, hallar los instrumentos para describirla y efectuar una relectura dentro de este nuevo contexto de las relaciones cambiantes del hombre con la naturaleza son los problemas cruciales de nuestra época (Prigogine, 1997, p.

48).

De Morin se toman las aproximaciones conceptuales que hace en La introducción del pensamiento complejo, pero cabe anotar que él dedica buena parte de su producción a la conceptualización del término asociado a su idea de pensamiento complejo. Estas son sus aproximaciones:

A primera vista, la complejidad es un tejido (complexus: lo que está tejido en conjunto) de constituyentes heterogéneos inseparablemente asociados: presenta la paradoja de lo uno y de lo múltiple. Al mirar con más atención, la complejidad es, efectivamente, el tejido de eventos, acciones, interacciones, retroacciones, determinaciones, azares, que constituyen nuestro mundo fenoménico. Así es que la complejidad se presenta con los rasgos inquietantes de lo enredado, de lo inextricable, del desorden, la ambigüedad, la incertidumbre... (Morin, 1996, p. 32).

Pero para Morin, también es

[...] un fenómeno cuantitativo, una cantidad extrema de interacciones e interferencias entre un número muy grande de unidades. [...]

Pero la complejidad no comprende solamente cantidades de unidades en interacciones que desafían nuestras posibilidades de cálculo, comprenden también incertidumbres, indeterminaciones, fenómenos aleatorios. En un sentido, la complejidad siempre está asociada con el azar. [...]

Pero la complejidad no se reduce a la incertidumbre, es la incertidumbre en el seno de los sistemas ricamente organizados. (Morin, 1996, p. 59-60).

Autores más próximos al trabajo en el campo de la educación se ubican en un terreno más amplio y, en un estrecho vínculo con el trabajo de Morin, asumen algunas definiciones del término.

Lo complejo se define o se aproxima a lo desconocido, a lo oscuro, al desorden, a lo incierto, a la antinomia y a lo dialéctico; eso es, en suma, la ruina de la física clásica y de la ciencia asentada en el método científico. La complejidad es, sin duda, un nuevo formato conceptual que debe propiciar una nueva forma de comprender $y$ explicar la realidad. En este sentido, diríamos 
que se trata de un sistema que se ve desbordado por la propia complejidad sistémica, por lo que, el sistema así considerado, se reconvierte en el elemento crítico de la teoría de sistemas. [...] (Colom, 2006, p. 19).

En términos comunes o triviales lo complejo se asocia generalmente a la idea de lo complicado, de algo enmarañado compuesto de múltiples aspectos y que es de difícil comprensión. En el vocabulario filosófico, lo complejo, como sustantivo, se aplica sólo a un conjunto de elementos de cualquier naturaleza (física, biológica, social, antropológica, psíquica, etc.) unidos en un todo orgánico (Foulquié, 1967).Y es que, en principio, la etimología de la palabra proviene del latín complexus, que significa aquello que está entretejido o tejido en conjunto.

Como adjetivo, dicho término califica a lo que contiene a un número grande de elementos de diferentes especies, a lo que forma un todo que, de ordinario, presenta aspectos diversificados. Así, se califica como "complejos" a fenómenos, situaciones, comportamientos, procesos, estructuras, etc., aunque en cada caso sea necesario precisar el sentido en que estos son complejos. [...] No es, entonces, simple multiplicidad, sino multiplicidad organizada. (Ochoa, 2006, p. 70).

Como pudo evidenciarse, la complejidad no puede ni entenderse ni asumirse desde una sola dimensión por cuanto - por una parte- las ciencias de la complejidad no se enmarcan en verdades definitivas y, por el contrario, transitan por el terreno de la construcción y reconstrucción permanentes y -por otra parte- el término se adapta a las condiciones que el fenómeno, el comportamiento o el sistema van evidenciando, cabe aclarar, eso sí, que la idea de lo complejo siempre implicará la idea de la multiplicidad, de la discontinuidad y de la interrelación o el entretejido que siempre estará cercano del caos, del cambio, de la transformación y que es allí donde las ciencias de la complejidad encuentran su objeto de estudio y discusión.

\section{Complejidad, pensamiento complejo y educación}

Como se ha dicho líneas atrás, la revisión que se ofrece en este documento se refiere al tema de complejidad y educación; sin embargo, cabe resaltar que es reconociendo la complejidad como método que esa relación se ha dado, posiblemente porque la educación -y todo lo que concierne a ella- ha estado limitada al campo de la acción más que al de la teorización, a pesar de los esfuerzos que han hecho autores como Not, Piaget, Colom y Melich, Dewey y Jerome Bruner, entre otros, por avanzar en la construcción teórica de este campo de las ciencias humanas. Esta es la razón por la cual, al parecer, su ubicación dentro de las ciencias de la complejidad responde a esa idea de práctica y de acción sobre el mundo y, en esa medida la literatura que hay al respecto se produce más en estos campos que en los de la teorización.

La revisión de la literatura conduce casi irremediablemente a un autor y a una corriente -si se le puede llamar así- que casi de manera exclusiva se encuentra en el origen de todos los trabajos que se han hecho en torno a complejidad y educación. El autor es Edgar Morin y el campo en el que se ha movido con mayor preponderancia es el del pensamiento complejo. Quizá la razón de interés en esta perspectiva radique en que, como bien lo dice Maldonado, la complejidad desde Morin responde a la lógica de una complejidad como método que es manifiestamente popular en la medida en que, además de utilizar un lenguaje que no es el que marca el camino hacia la complejidad -que no es ni matemático, ni biológico, ni físico-, la gran dificultad que tiene el pensamiento complejo es que se reduce a una hermenéutica y, de alguna manera la lógica de la complejidad implicaría el estudio de lo real y de lo posible y sobre todo de lo posible como fundamento de lo real y en eso la propuesta de Morin no alcanzaría el objetivo (Maldonado, 1999, pp. 13-25).

Sin embargo, y a pesar de la contundencia de esta posición enunciada, es especialmente desde Morin que se estructuran la cantidad de estudios y reflexiones que se han adelantado en educación; su obra se enuncia como pilar del trabajo educativo en muchas regiones del mundo y, muy especialmente, en Latinoamérica.

Cabe aclarar, entonces, que aunque es factible que existan estudios que aborden la educación desde las 
ciencias de la complejidad en un plano teórico, el trabajo adelantado en esta revisión ubica el fundamento de la relación complejidad y educación en los trabajos de Morin y en su idea de que al presentarse la complejidad "con los rasgos inquietantes de lo enredado, de lo inextricable, del desorden, la ambigüedad, la incertidumbre..." (Morin, 1996, p.32) el conocimiento enfrenta la necesidad de poner orden, de reducir la incertidumbre de clarificar y en esa tarea se corre el riesgo de volverse ciegos. Por esta razón, el pensamiento complejo debe "afrontar lo entramado, [...] la solidaridad de los fenómenos entre sí,la bruma, la incertidumbre, la contradicción [para lo cual se pueden] diseñar algunos de los útiles conceptuales, algunos de los principios, para esa aventura" (Morin, 1996, p. 33).

Estos principios de los que habla son, quizá, el aspecto de su propuesta que más se cita en las publicaciones que acerca de complejidad y educación se han venido haciendo desde finales del siglo pasado y que, al decir de Morin "pueden ayudarnos a pensar la complejidad” (1996, p. 105): el principio dialógico, el cual permite mantener la dualidad en el seno de la unidad; el principio de recursividad organizacional y que se podría asimilar al concepto de autoorganización; y, el principio hologramático en el que el menor punto de la imagen del holograma contiene la casi totalidad de la información (p. 106-108).

Otro punto de referencia obligado para quienes trabajan esta relación complejidad educación es el documento que elaboró el autor para Unesco, en el que plantea los sietes saberes fundamentales que la "educación del futuro" debería tratar en cualquier sociedad y en cualquier cultura.

Con estos dos referentes básicos, con la claridad de que el tema no se agota en estas líneas y con la tarea urgente de adentrarse en el estudio de los fenómenos, estructuras y sistemas que la educación, como ejemplo propicio de un sistema abierto, impredecible, nolienal, autoorganizado e irreversible, se referencian a continuación algunos de los trabajos que -con todas las dificultades que, como ya se ha dicho, enfrenta el campo de la educación al priorizar su acción sobre el mundo dejando de lado muchas veces la construcción teórica de su realidad, constituyen un aporte para la discusión y allanan un camino posible para entender su complejidad.

a. La obra de Morin, especialmente la publicada a partir de la segunda mitad de la década del noventa $y$, como se ha planteando anteriormente, la concepción de pensamiento complejo, los principios que ayudarían a comprender el mundo desde ese pensamiento y, especialmente, los saberes que le definen un horizonte a la educación en el mundo.

b. Algunos trabajos realizados por investigadores españoles que, fundamentados parcialmente en Morin, se arriesgan a una producción algo más teórica. Se tiene aquí el trabajo de Santos Rego y Guillaumín Tostado, editores y autores de un trabajo que mira la educación de manera compleja en sus diferentes ángulos y relaciones, lo cual les implica entrelazar los saberes que las neurociencias, las ciencias sociales y las ciencias cognitivas, entre otras, han producido en torno a temas como el aprendizaje, la pedagogía, el conflicto, la discriminación y en general todos aquellos aspectos del sistema que confluyen en un mismo tiempo y lugar y que cambian con la facilidad que les marca un atractor. El trabajo de estos editores se organiza en dos partes: la primera se desarrolla en el marco de la disquisición teórica y la segunda en el de la aplicación. Otro trabajo semejante a este, pero con menos pretensiones, se recoge en el libro de Rafael Yus Ramos quien aventura la relación educación y complejidad alrededor de la educación holística como una forma de "hacer frente al paradigma de la simplicidad, anteponiendo un paradigma de la complejidad, [que se] alcanza con el adiestramiento del pensamiento sistémico o complejo" (Yus Ramos, 2001, p. 167).

c. Los trabajos adelantados en Latinoamérica en cuya formulación inicial participó activamente Colombia, al finalizar el siglo anterior y de los cuales cabe resaltar el Manual de iniciación pedagógica al pensamiento complejo y la compilación de Sergio González Moena, que se publica dos años después de la visita de Morin a Colombia en 1997. El primero de estos trabajos constituye 
en su mayor parte una revisión de lo que ha sido la evolución de la complejidad, sus orígenes, las teorías que se han producido alrededor del tema, así como la relación de la complejidad con el tema educativo, especialmente, en el que estas ciencias han trabajado arduamente: la transdisciplinariedad. El segundo constituye un trabajo de reflexión que compila las ideas básicas de Morin acerca de la complejidad, una especie de diálogo entre los pensamientos de Edgar Morin, Humberto Maturana y Christian Parker, alrededor del problema del conocimiento y un ejercicio de análisis acerca de la complejidad y los saberes en educación superior.

d. Siguiendo con Latinoamérica, pero no en trabajos regionales sino en cada país y con difusión a través de revistas digitales, llama la atención el trabajo permanente que ha sostenido la Red Mexicana de Pensamiento Complejo (www.unla. edu.mx) originada en la Cátedra Itinerante de Unesc Edgar Morin, y de la cual ya circulan diez boletines, en los cuales se registra al menos un artículo referido a la educación. De igual manera, la página www.pensamientocomplejo.com.ar que

\section{Bibliografía}

Binder, Philippe (1999). Cuatro versiones de la complejidad. En: Visiones sobre la complejidad. 1a. edición. Bogotá: Ediciones el Bosque.

Carreras, A.; Escorihuela, J.I. y Requejo, A. (eds.) (1990). Azar, caos e indeterminismo. $1^{\text {a }}$. edición. Zaragoza: Universidad de Zaragoza.

Colom Cañellas, Antoni J. (2006). Complejidad y teoría del caos en educación. En: Avances en complejidad y educación: teoría y práctica. 1a. edición. Barcelona: Ediciones Octaedro, S.L.

González Moena, Sergio (comp.) (1997). Pensamiento complejo. En torno a Edgar Morin, América Latina $y$ los procesos educativos. Colombia: Cooperativa Editorial Magisterio.

Maldonado, Carlos Eduardo. (1999). Esbozo de una filosofía de la lógica de la complejidad. En: Visiones aunque tiene como tema central la educación, dedica algunos de sus trabajos al tema, de hecho la biblioteca virtual que ofrece tiene varios títulos referidos a educación. La Revista Contexto Educativo de Argentina, (www.contexto-educativo. com.ar) que ocasionalmente ofrece artículos sobre el tema y muchas otras revistas digitales que en Latinoamérica han centrado su atención en el tema de la complejidad y la educación.

Son muchas las deudas que quedan en este recorrido, pero quizá la más grande de ellas es la que tiene la educación con el estudio de su realidad en el marco de las ciencias de la complejidad que supere la idea de que sólo lo práctico, sólo lo aplicado, sólo la mera realidad es suficiente para comprender y transformar el mundo. Esta tarea pendiente en esencia no ha cambiado, en la medida en que toda la historia de la educación y la pedagogía ha enfrentado, sin superarlo, su condición de ser instrumento para, situación que la ha condenado a responder siempre a lo que otras ciencias, otros poderes, otros pensamientos quieran de ella. La oportunidad de pensarse en las ciencias de la complejidad, es una oportunidad a la que no se puede dar la espalda.】

sobre la complejidad. 1ª edición. Bogotá: Ediciones el Bosque.

Maldonado, Carlos Eduardo. (2005). Ciencias de la complejidad: Ciencias de los cambios súbitos. Odeon. Observatorio de economía y operaciones numéricas, 2, 85-125.

Maldonado, Carlos Eduardo. (2005). Termodinámica y complejidad. $1^{\text {a }}$. edición. Bogotá: Universidad Externado de Colombia.

Maldonado, Carlos Eduardo. (2005a). Complejidad de las ciencias y ciencias de la complejidad. $1^{\text {a }}$. edición. Bogotá: Universidad Externado de Colombia.

Moreno, Juan Carlos, et. ál. (2002). Manual de iniciación pedagógica al pensamiento complejo. Bogotá: Instituto Colombiano de Fomento para la Educación Superior, ICFES-UNESCO, Corporación para el desarrollo Complexus. 
Morin, Edgar. (1996). Introducción al pensamiento complejo. Barcelona: Editorial Gedisa.

Morin, Edgar. (2001). Los siete saberes necesarios. Bogotá: Cooperativa Editorial Magisterio.

Morin, Edgar. (2004). La epistemología de la complejidad. Gaceta de Antropología. o 20. Texto 20-02. Consultado el 25 de mayo de 2007 en: http://www. ugr.es/ pwlac/G20_02Edgar_Morin.html En www. pensamientocomplejo.com.ar.

Ochoa Contreras, Octavio. (2006). Apuntes para una educación universitaria del siglo XXI desde la perspectiva del pensamiento complejo. En: Avances en complejidad y educación: teoría y práctica. $1^{\text {a }}$. edición. Barcelona: Ediciones Octaedro, S.L.

Prigogine, ILSA. (1997). ¿Tan solo una ilusión? Una exploración del caos al orden. 4a edición. Barcelona: Tusquets Editores.

Yus Ramos, Rafael. (2001). Educación Integral. Una educación holística para el siglo XXI. Vol. II. Bilbao: Editorial Desclée de Broker.

www.unla.edu.mx

www.contexto-educativo.com.ar

www.pensamientocomplejo.com.ar 
